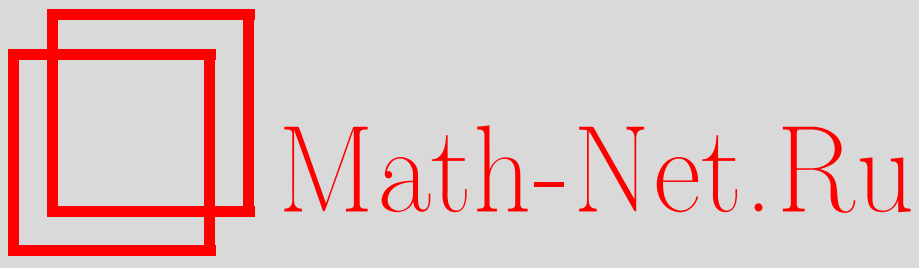

В. В. Кабанин, Л. В. Согоцьян, И. Г. Овчинников, Ползучесть и долговечность стержня, взаимодействующего с жидким натрием, Вестн. Сам. гос. техн. ун-та. Сер. Физ.-мат. науки, 2007, выпуск 1(), 50-55

DOI: https://doi.org/10.14498/vsgtu487

Использование Общероссийского математического портала Math-Net.Ru подразумевает, что вы прочитали и согласны с пользовательским соглашением http://www . mathnet.ru/rus/agreement

Параметры загрузки:

IP: 54.166 .219 .16

26 апреля 2023 г., 16:29:51

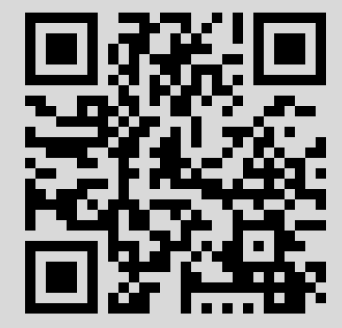




\section{ПОЛЗУЧЕСТЬ И ДОЛГОВЕЧНОСТЬ СТЕРЖНЯ, ВЗАИМОДЕЙСТВУЮЩЕГО С ЖИДКИМ НАТРИЕМ}

Предложена математическая модель, описывающая ползучесть и длительную прочность сталей перлитного класса с агрессивной средой - жидким натрием. В качестве базовой теории использовались кинетические уравнения Ю.Н. Работнова. В совокупности с уравнениями диффузии, описываюшими кинетику механических характеристик материала, предложен устойчивый численный алгоритм решения задачи. Приведень результаты расчета напряженно-деформированного состояния стержня в жидком натрии. Выполнена проверка адекватности расчетной модели экспериментальным данным.

1. Рассмотрим основные соотношения математической модели, описывающей взаимодействие сталей перлитного класса с жидким натрием. При построении модели была принята следующая упрощенная картина явлений, протекающих при работе конструктивного элемента в жидком натрии. При одновременном присутствии элементов из аустенитных и перлитных сталей в контуре системы перлитные стали постепенно обезуглероживаются, в результате чего содержание углерода в конструктивном элементе с течением времени изменяется, уменьшаясь по направлению к поверхности, контактирующей с натрием. Если элемент конструкции находится в напряженном состоянии, то в нем протекают процессы ползучести, релаксации и накопления повреждений. Обезуглероживание сильно влияет на кинетику этих процессов, ускоряя их. В случае, когда элемент конструкции находится в горячей зоне контура, имеет место термический перенос массы, приводящий к разрыхлению поверхности и уменьшению толщины элемента. Такая упрощенная физическая модель позволяет воспользоваться для построения математической модели методами механики сплошной среды, в частности, теорией структурных параметров Ю. Н. Работнова [1]. При этом состояние и поведение моделей будет характеризоваться не только механическими, но и физико-химическими параметрами. В систему определяющих параметров включены: напряжение $\sigma$, деформация $\varepsilon$, температура $T$, параметр поврежденности П, а также дополнительный параметр, характеризующий интенсивность воздействия жидкометаллической среды на характеристики ползучести и длительной прочности в различных точках по толщине конструктивного элемента. Эксперименты свидетельствуют, что изменение указанных характеристик коррелирует с изменением концентрации углерода, поэтому в качестве дополнительного параметра оказалось возможным принять концентрацию $C$ углерода в точке конструкции. Закон распределения концентрации углерода по объему конструктивного элемента находится из решения уравнения диффузии. Причем коэффициент диффузии углерода $D$ в материале может зависеть от уровня напряжений, поврежденности и температуры в рассматриваемой точке.

При построении модели были учтены следующие эффекты [2]: а) обезуглероживание приводит к ускорению ползучести; б) на кривых ползучести могут наблюдаться все три стадии ползучести (неустановившаяся, установившаяся и ускоренная); в) обезуглероживание приводит к увеличению деформации при разрушении для одного и того же уровня напряжений; г) увеличение уровня напряжений снижает эффект увеличения скорости ползучести от обезуглероживания; д) допускается, что обезуглероживание приводит к смещению и повороту кривых $p^{*}(\sigma)$, где $p^{*}$ - предельная деформация ползучести; е) обезуглероживание приводит к значительному сокращению долговечности; ж) допускается, что обезуглероживание приводит к параллельному смещению кривых длительной прочности в логарифмических координатах.

При описании поведения материала с учетом влияния обезуглероживания полагалось, что полная деформация $\varepsilon$ складывается из упругой и деформации ползучести $p$ :

$$
\varepsilon=\sigma / E+p
$$

где $E(C)$ - модуль упругости, величина которого зависит от содержания углерода в материале. Уравнения ползучести принимались в виде, описывающем все три стадии ползучести:

$$
\begin{gathered}
\frac{d p}{d t}=A p^{-\alpha}\left(\frac{\sigma}{1-\mu \Pi}\right)^{k}, p(0)=0, p\left(t_{\mathrm{p}}\right)=p^{*} ; \\
\frac{d \Pi}{d t}=\frac{B \sigma^{n}}{(1-\Pi)^{n+S}}, \Pi(0)=0, \Pi\left(t_{\mathrm{p}}\right)=1 .
\end{gathered}
$$


Здесь $t_{\mathrm{p}}$ - время до разрушения; $A, \alpha, k, B, n, S, \mu-$ коэффициенты; П - параметр поврежденности.

Влияние обезуглероживания на кинетику деформирования и разрушения учитывалось зависимостью коэффициентов уравнений (2) и (3) от концентрации углерода. В дальнейшем в работе детально исследовался случай кинетических уравнений (2), (3) при $\mu=1$. Тогда интегрированием (2) и (3) при значениях коэффициентов, соответствующих определенному уровню $C$ и $\sigma=$ const, получается уравнение ползучести

$$
\left.p=\left(\frac{A(\alpha+1) \sigma^{k-n}}{B(n+S+1-k)}\right) \cdot\left(1-\left(1-B(n+S+1) \sigma^{n} t\right)^{\frac{n+S+1-k}{n+S+1}}\right)\right)^{\frac{1}{\alpha+1}}
$$

и уравнение накопления повреждений

$$
\Pi=1-\left(1-B \cdot(n+S+1) \cdot \sigma^{n} \cdot t\right)^{\frac{1}{n+S+1}} .
$$

Подставляя условия $p\left(t_{\mathrm{p}}\right)=p^{*}$ и $\Pi\left(t_{\mathrm{p}}\right)=1$ в (4) и (5), получим уравнение кривой длительной прочности

$$
t_{\mathrm{p}}=1 /\left(B(n+S+1) \sigma^{n}\right)
$$

и зависимость деформации при разрушении от напряжения вида

$$
p^{*}=\left(A(\alpha+1) \sigma^{k-n} /(B(n+S+1-k))\right)^{\frac{1}{\alpha+1}} .
$$

Явный вид зависимостей коэффициентов $\mathrm{A}(C), B(C), \alpha(C), k(C), n(C), S(C)$, а также $E(C)$ определяется по их значениям, найденным с помощью формул (4), (6), (7) по опытным данным для материала с разными уровнями $C$. Указанный способ моделирования требует значительного количества экспериментальных данных.

Иной подход к учету влияния обезуглероживания на процессы деформирования и разрушения заключается в использовании подобия кривых ползучести и длительной прочности материала при разных концентрациях углерода и уровнях напряжений. Это позволяет представить основные соотношения, описывающие поведение материала в виде:

$$
\begin{gathered}
\frac{d p}{d t}=A_{\varphi} p^{-\alpha_{0}}\left(\frac{\sigma}{1-\mu \cdot \Pi}\right)^{k}, p(0)=0, p\left(t_{\mathrm{p}}\right)=p^{*}, \\
\frac{d \Pi}{d t}=\frac{B_{\psi} \sigma^{n_{0}}}{(1-\Pi)^{n_{0}+S_{0}}}, \Pi(0)=0, \Pi\left(t_{\mathrm{p}}\right)=1,
\end{gathered}
$$

где

$$
\begin{aligned}
& A_{\varphi}=A_{0} \varphi^{k} \\
& B_{\psi}=B_{0} \psi^{k} .
\end{aligned}
$$

Здесь индекс «0» соответствует значениям коэффициентов для материала в исходном (необезуглероженном) состоянии; $\varphi(C), \psi(C)$ - некоторые функции, учитывающие влияние уровня обезуглероживания на ползучесть и накопление повреждений, причем $\varphi>1, \psi>1$. Интегрируя (8) и (9) при $\sigma=$ const, $\mu=1$, постоянных значениях коэффициентов и используя условия $p\left(t_{\mathrm{p}}\right)=p^{*}, \Pi\left(t_{\mathrm{p}}\right)=1$, получим:

$$
\begin{gathered}
t_{\mathrm{p}}=1 /\left(B_{\psi}\left(n_{0}+S_{0}+1\right) \sigma^{n_{0}}\right), \\
p^{*}=\left(A_{\varphi}\left(\alpha_{0}+1\right) \sigma^{k_{0}-n_{0}} /\left(B_{\psi}\left(n_{0}+S_{0}+1-k_{0}\right)\right)\right)^{\frac{1}{\alpha_{0}+1}} .
\end{gathered}
$$

2. Стержневые элементы используются в качестве испытательных образцов при опытах по определению характеристик длительной прочности и ползучести материалов как в нейтральной, так и в жидкометаллической средах. На рис. 1 показаны некоторые программы нагружения опытных образцов, которые используются при испытаниях конструкционных перлитных сталей, предназначенных для эксплуатации в жидком натрии ( $N$ - растягивающая нагрузка).

Рассмотрим расчет напряженно-деформированного состояния и долговечности круглого стержня, нагружаемого по программе $1, a$. Считаем, что стержень предварительно прогрет до температуры $T$, затем к нему прилагается растягивающая сила $N$, после чего он помещается в жидкометаллическую среду. На этапе силового нагружения при $t=0$ решается упругая задача: и находятся напряжение и деформация: 


$$
\sigma=N /\left(\pi R^{2}\right), \quad \varepsilon=\sigma / E_{0},
$$

где $N$ - растягивающая сила, $E_{0}$ - модуль упругости необезуглероженного материала. Далее происходит процесс деформирования во времени, сопровождающийся обезуглероживанием материала стержня. Для начала вычислительного процесса необходимо ввести дополнительный этап расчета, в процессе которого возмущается граничная концентрация углерода. Необходимость этого этапа объясняется особенностью численного решения задачи диффузии, так как «мгновенное» установление граничного условия приводит к резкой неоднородности механических свойств материала по поперечному сечению стержня, из-за чего скорости деформирования в разных точках сечения существенно отличаются, в результате задача определения напряженно-деформированного состояния стержня становится неустойчивой. С аналогичными проблемами столкнулись авторы ряда работ, в которых (с целью их устранения) вводился этап установления граничного условия. Вычислительный эксперимент, проведенный при разработке описываемой здесь методики, выявил необходимость введения такого этапа для расчета стержневого элемента, обезуглероживающегося в жидком натрии. В данном случае уравнение диффузии будет иметь вид

$$
\frac{\partial\left(C_{0}-C\right)}{\partial t}=D\left(\frac{\partial^{2}\left(C_{0}-C\right)}{\partial^{2} r}-\frac{1}{r} \cdot \frac{\partial\left(C_{0}-C\right)}{\partial r}\right),
$$

с начальным и граничным условиями:

$$
\left.\left(C_{0}-C\right)\right|_{t=0}=0,\left.\quad\left(C_{0}-C\right)\right|_{r=R}=C_{\infty}=\text { const, }
$$

где разность $\left(C_{0}-C\right)$ характеризует потери концентрации углерода в точке элемента конструкции.
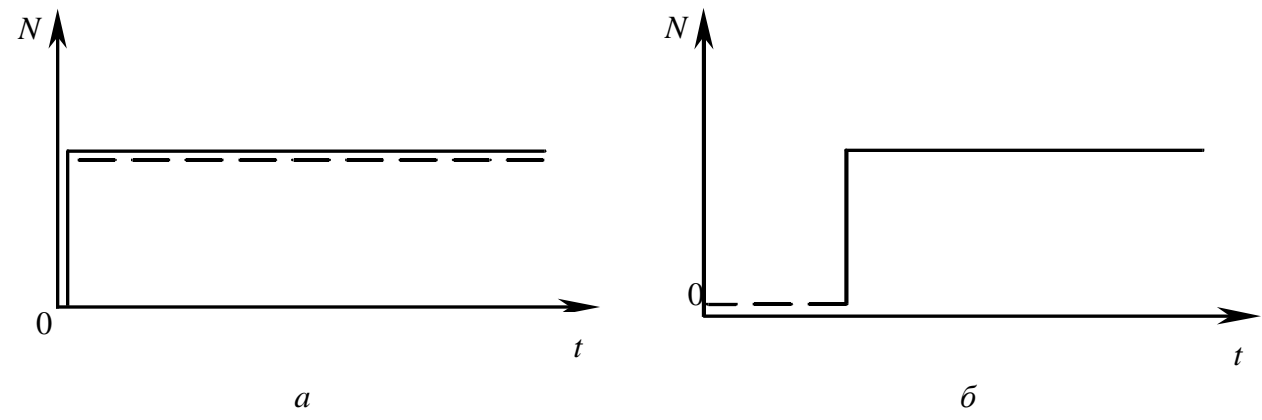

Р и с. 1. Программы нагружения опытных образцов в жидком натрии

Для получения физических соотношений оказывается удобным использовать метод последовательных возмущений параметров. За шаг по времени $\Delta t$ полная деформация изменится на величину

$$
\Delta \varepsilon=\frac{\partial \varepsilon}{\partial \sigma} \Delta \sigma+\frac{\partial \varepsilon}{\partial E} \Delta E+\frac{\partial \varepsilon}{\partial p} \Delta p
$$

Учитывая, что $\frac{\partial \varepsilon}{\partial \sigma}=\frac{1}{E} ; \frac{\partial \varepsilon}{\partial E}=-\frac{\sigma}{E^{2}} ; \frac{\partial \varepsilon}{\partial p}=1$, получим для приращения напряжения соотношение

$$
\Delta \sigma=E \cdot(\Delta \varepsilon-\Delta p)+\frac{\sigma}{E} \Delta E .
$$

Так как на этапе деформирования во времени приращение растягивающей силы не происходит, можно записать:

$$
\Delta N=\int_{F} \Delta \sigma d F=2 \pi \int_{0}^{R} \Delta \sigma r d r=0
$$

С учетом (18) имеем

$$
\Delta \varepsilon=\frac{\int_{0}^{R} E \Delta p r d r-\int_{0}^{R} \frac{\sigma}{E} r d r}{\int_{0}^{R} E r d r} .
$$


Величина приращения деформации ползучести $\Delta p_{\text {u }}$ определяется интегрированием уравнений (2) и (3).

При решении задачи Коши для уравнений ползучести и накопления повреждений возникает проблема вычисления правых частей уравнений, которые характеризуют напряженнодеформированное состояние элемента на данном временном шаге $\Delta t$ и зависят от искомых функций $p$ и $П$, рассматриваемых на этом же шаге по времени. Для преодоления этой трудности, обычно предполагают, что в пределах временного интервала $\Delta t$ правые части дифференциальных уравнений практически стационарны. Это позволяет ограничиться итерационным алгоритмом решения задачи Коши, основанном на использовании простых явных и неявных схем интегрирования [3, 4]. Воспользовавшись способом, предложенным Н. Н. Калиткиным [3], преобразуем исходную систему (2), (3) так, чтобы разделить переменные; в результате получим:

$$
\begin{aligned}
& p^{\alpha} d p=A\left(\frac{\sigma}{1-\Pi}\right)^{k} d t, \\
& (1-\Pi)^{n+S} d \Pi=B \sigma^{n} d t .
\end{aligned}
$$

Далее, интегрируя (21) и (22) в пределах шага $\Delta t$ на временном интервале $t_{i} \leq t \leq t_{i+1}$, имеем:

$$
\begin{aligned}
& \int_{P_{i}}^{P_{i+1}} p^{\alpha} d p=\int_{t_{i}}^{t_{i+1}} A\left(\frac{\sigma}{1-\Pi}\right)^{k} d t, \\
& \int_{\Pi_{i}}^{\Pi_{i+1}}(1-\Pi)^{n+S} d \Pi=\int_{t_{i}}^{t_{i+1}} B \sigma^{n} d t .
\end{aligned}
$$

Как показали численные эксперименты, для получения сходящегося итерационного процесса, достаточно аппроксимировать интегралы в (23) и (24) простыми квадратурными формулами, самой простой из которых оказывается формула левых прямоугольников, приводящая к явной разностной схеме.

Изложенный подход налагает ограничение на величину шага по времени, поэтому будем использовать специальную процедуру выбора шага. Для этого задаем предельные величины изменения параметров поврежденности $\Delta \Pi_{\max }$ и деформаций ползучести $\Delta p_{\max }$ за один шаг по времени $\Delta t$ по каждой из точек сетки, соответственно исходя из условий предельного изменения параметров П и $p$ :

$$
\begin{aligned}
& \Delta t_{\Pi_{i}}=\frac{\left(1-\Pi_{i-1}\right)^{n+S} \Delta \Pi_{\max }}{B_{i-1} \sigma_{i-1}^{n}}, \\
& \Delta t_{P_{i}}=\frac{\left(1-\Pi_{i-1}\right)^{k} P_{i-1}^{\alpha} \Delta p_{\max }}{A_{i-1} \sigma_{i-1}^{k}} .
\end{aligned}
$$

При использовании явной схемы для решения уравнения диффузии необходимо также учесть ограничение на шаг по времени, налагаемое условием устойчивости разностной схемы.

На основе вышеизложенного был разработан следующий алгоритм расчета.

1. В момент $t=0$ производится силовое нагружение и определяются напряжение $\sigma$ и деформация $\varepsilon$, задается начальный шаг по времени $\Delta t$.

2. Задается шаг возмущения граничной концентрации $\Delta C$.

3. Решается уравнение диффузии, находится массив концентраций углерода С.

4. Рассчитываются коэффициенты $A(C)$ и $B(C)$ модели (2), (3).

5. Решаются уравнения ползучести (2) и накопления повреждений (3) с использованием формул (23) и (24), после чего по формулам:

$$
\Delta p_{i}=p_{i}-p_{i-1}, \Delta \Pi_{i}=\Pi_{i}-\Pi_{i-1}
$$

находятся массивы приращений деформаций ползучести и приращений поврежденностей.

6. Производится проверка по каждой из точек разбиения выполнения условий

$$
\begin{aligned}
\Delta p_{i} & \leq \Delta p_{\max }, \\
\Delta \Pi_{i} & \leq \Delta \Pi_{\max } .
\end{aligned}
$$

В случае их невыполнения шаг $\Delta t$ уменьшается и происходит возврат к шагу 3.

7. Определяется массив модулей упругости $E(C)$ и его приращение $\Delta E=E_{i}-E_{i-1}$.

8. Численным интегрированием находятся интегралы, входящие в выражение (20).

9. Определяется приращение полной деформации $\Delta \varepsilon$ по формуле (20). 
10.Из выражения (18) находится массив приращений напряжений $\Delta \sigma$.

11.Проверяется условие по ограничению относительных величин возмущений напряжений по каждой из точек дискретизации. В случае невыполнения шаг $\Delta t$ уменьшается и производится возврат к шагу 3.

12.Находится массив напряжений $\sigma$ по формуле $\sigma_{i+1}=\sigma_{i}+\Delta \sigma$.

13. Производится проверка на монотонность изменения поля напряжений. В случае невыполнения шаг $\Delta t$ уменьшается и производится возврат к шагу 3.

14. Проверяются некоторые условия завершения программы и обработка прерываний. Делается подготовка к следующему шагу по времени и выбирается его величина по условиям (25) и (26) и условию устойчивости разностной схемы решения уравнения диффузии

$$
\Delta t \leq\left(\frac{\Delta r^{2}}{4 D}\right) .
$$

15. Производится проверка установления граничных условий для уравнения диффузии; в случае достижения равновесного значения $C_{\infty}$ дальнейшие вычисления производятся с шага 3 , иначе делается очередное возмущение граничного условия - на величину $\Delta C$ (шаг 2).

Описанный алгоритм реализован в виде программного комплекса, с использованием которого выполнена серия численных экспериментов. Модель дает хорошее приближение к экспериментальной кривой и описывает в пределах разброса опытных данных время до разрушения и деформацию при разрушении как в нейтральной среде, так и при обезуглероживании в жидком натрии (см. таблицу).

Сравнение результатов расчета стержня в нейтральной среде и жидком натрии с опытными данными

\begin{tabular}{|c|c|c|c|c|c|c|c|c|}
\hline $\begin{array}{c}\sigma, \\
\text { МПа }\end{array}$ & \multicolumn{4}{|c|}{ на воздухе } & \multicolumn{4}{|c|}{ в жидком натрии } \\
\hline & 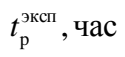 & $t_{\mathrm{p}}^{\mathrm{pacu}}$, час & 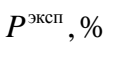 & $P^{\text {расч }}, \%$ & 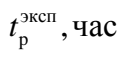 & $t_{\mathrm{p}}^{\text {расч }}$, час & 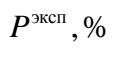 & 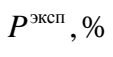 \\
\hline 100 & 890 & 870 & 34 & 33 & 750 & 730 & 40 & 44 \\
\hline 80 & 5150 & 4970 & 24 & 26 & 3100 & 2930 & 34 & 36 \\
\hline
\end{tabular}

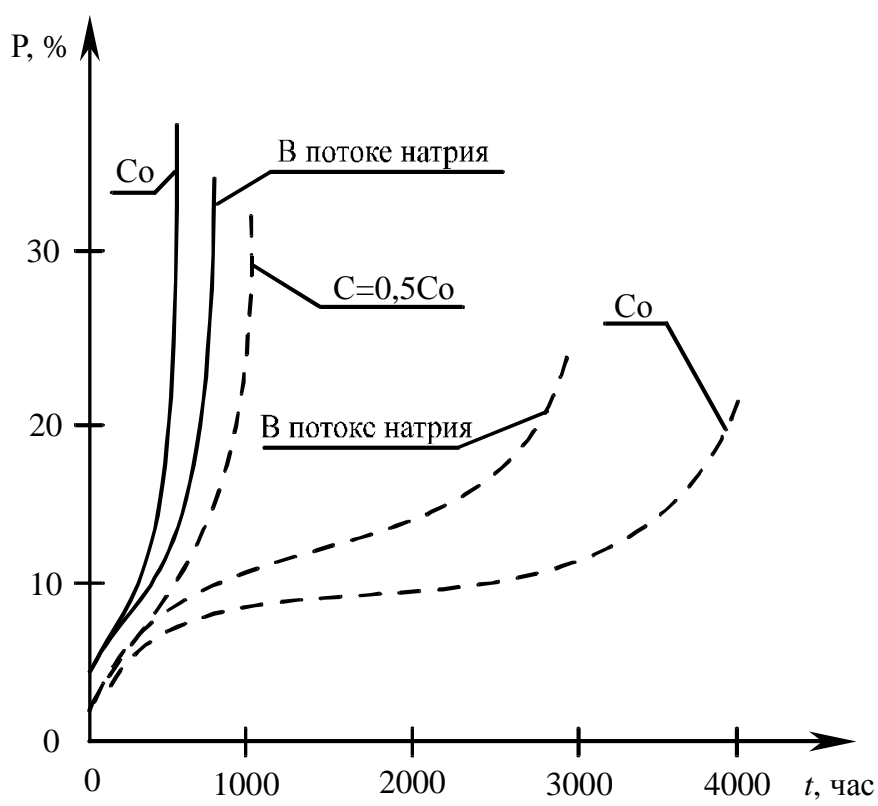

Р и с. 2. Кривые ползучести на воздухе и в жидком натрии: сплошные линии - $\sigma=100$ МПа; штриховые ли-

$$
\text { нии }-\sigma=86 \text { МПа }
$$

Кривая ползучести для образца (см. рис. 2), испытанного в жидком металле, лежит между кривыми ползучести для образцов, испытанных в нейтральной среде, но имеющих разную концентрацию $\left(C=C_{0}\right.$ и $\left.C=0,5 C_{0}\right)$ (что соответствует гипотезе, заложенной при разработке модели).

На рис. 3. показаны профили концентрации углерода в различные моменты времени. Процесс обезуглероживания приводит к значительному перераспределению напряжений, причем напряжения в отдельных частях сечения могут вначале возрастать, а затем убывать.

Это иллюстрирует рис. 4. Как видно из рис.унка 5, разрушение происходит в точке, лежащей в частично обезуглероженной зоне, Анализ показывает, что разрушение наступает вследствие накоп-

ленной в точке поврежденности за весь период жизни образца. При этом напряжения в этой точке в момент разрушения не являются максимальными. Отсюда можно сделать вывод, что максимальные напряжения не могут выступать в роли критерия разрушения - важен весь процесс деформирования во времени и кинетика перераспределения напряжений из-за обезуглероживания. 


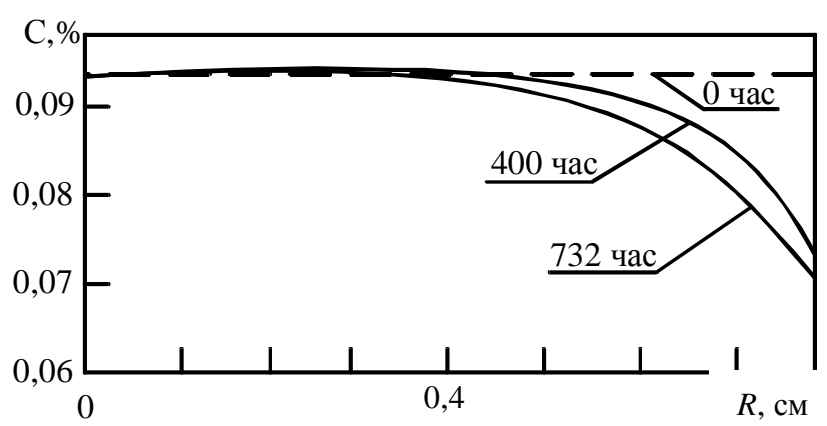

Р и с. 3. Эпюры концентрации углерода по радиусу стержня в разные моменты времени

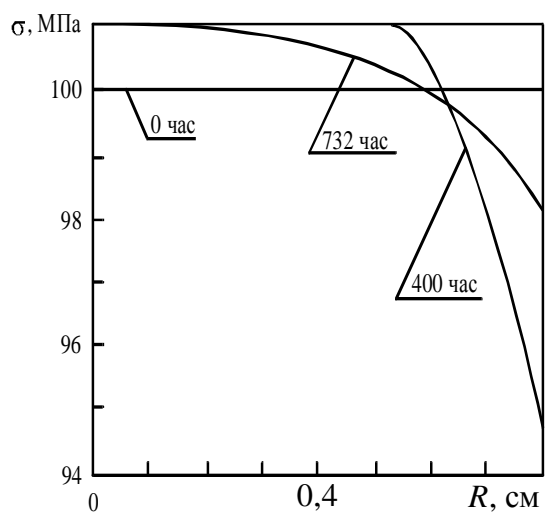

Р и с. 4. Изменение эпюр напряжений по сечению стержня в процессе обезуглероживания в жидком натрии

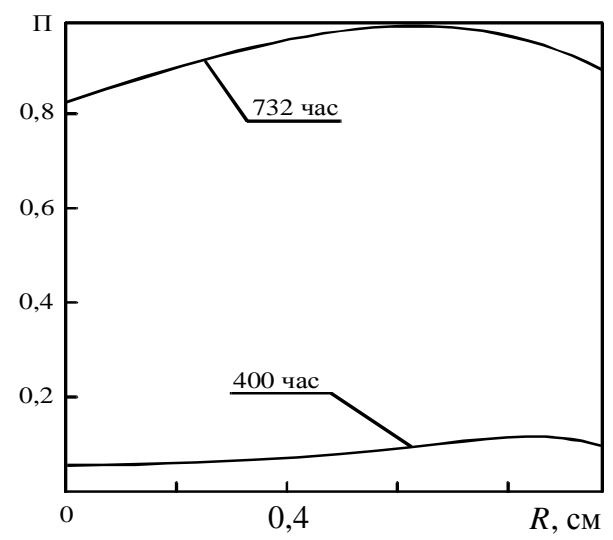

Р и с. 5. Эпюры поврежденности по сечению стержня в разные моменты времени

\section{БИБЛИОГРАФИЧЕСКИЙ СПИСОК}

1. Работнов Ю. Н. Ползучесть элементов конструкций. М.: Наука. 1966.752 с.

2. Овчинников И. Г., Северюхин Н. В. Построение модели деформирования и разрушения элемента конструкции, обезуглероживаемого в жидком металле // Сб.: Коррозия металлов и сплавов. Алма-Ата: КазПТИ, 1985. C. 81-87.

3. Калиткин Н. Н. Численные методы. М.: Наука, 1978. 512 с.

4. Форсайт Дж., Малькольм М., Моулер К. Машинные методы математических вычислений. М.: Мир, 1980. $279 \mathrm{c}$. 